\title{
Prolonged activation of IL-5-producing ILC2 causes pulmonary arterial hypertrophy
}

Masashi Ikutani, ${ }^{1,2}$ Koichi Tsuneyama, ${ }^{3,4}$ Makoto Kawaguchi, ${ }^{5}$ Junya Fukuoka, ${ }^{6}$ Fujimi Kudo, Susumu Nakae, ${ }^{7,8}$ Makoto Arita, , 8,9,10,11 Yoshinori Nagai, ${ }^{1,8}$ Satoshi Takaki, ${ }^{2}$ and Kiyoshi Takatsu, ${ }^{1,12}$

'Department of Immunobiology and Pharmacological Cenetics, Graduate School of Medicine and Pharmaceutical Science for Research, University of Toyama, Toyama, Japan. ²Department of Immune Regulation, The Research Centre for Hepatitis and Immunology, Research Institute, National Center for Global Health and Medicine, Chiba, Japan. ${ }^{3}$ Department of Diagnostic Pathology, Graduate School of Medicine and Pharmaceutical Sciences, University of Toyama, Toyama, Japan. ${ }^{4}$ Molecular and Environmental Pathology, Institute of Health Bioscience, The University of Tokushima Graduate School, Tokushima, Japan. ${ }^{5}$ Department of Diagnostic Pathology, Niigata Rosai Hospital, Japan Organization of Occupational Health and Safety, Niigata, Japan. ${ }^{6}$ Department of Pathology, Nagasaki University Graduate School of Biomedical Sciences, Nagasaki, Japan. 'Laboratory of Systems Biology, Center for Experimental Medicine and Systems Biology, The Institute of Medical Science, The University of Tokyo, Tokyo, Japan. ${ }^{8}$ JST, PRESTO, Saitama, Japan. ' ${ }^{2}$ Laboratory for Metabolomics, RIKEN Center for Integrative Medical Sciences (IMS), Kanagawa, Japan. ${ }^{10} \mathrm{Graduate}$ School of Medical Life Science, Yokohama City University, Kanagawa, Japan. "Division of Physiological Chemistry and Metabolism, Graduate School of Pharmaceutical Sciences, Keio University, Tokyo, Japan. ${ }^{12}$ Toyama Prefectural Institute for Pharmaceutical Research, Toyama, Japan.

\begin{abstract}
IL-33 is one of the critical cytokines that activates group 2 innate lymphoid cells (ILC2s) and mediates allergic reactions. Accumulating evidence suggests that IL-33 is also involved in the pathogenesis of several chronic inflammatory diseases. Previously, we generated an IL-5 reporter mouse and revealed that lung IL-5-producing ILC2s played essential roles in regulating eosinophil biology. In this study, we evaluated the consequences of IL-33 administration over a long period, and we observed significant expansion of ILC2s and eosinophils surrounding pulmonary arteries. Unexpectedly, pulmonary arteries showed severe occlusive hypertrophy that was ameliorated in IL5- or eosinophil-deficient mice, but not in Rag2-deficient mice. This indicates that IL-5-producing ILC2s and eosinophils play pivotal roles in pulmonary arterial hypertrophy. Administration of a clinically used vasodilator was effective in reducing IL-33-induced hypertrophy and repressed the expansion of ILC2s and eosinophils. Taken together, these observations demonstrate a previously unrecognized mechanism in the development of pulmonary arterial hypertrophy and the causative roles of ILC2 in the process.
\end{abstract}

Conflict of interest: JF is the representative director of Pathology Institute Corp and owns its stocks.

Submitted: October 7, 2016 Accepted: February 23, 2017 Published: April 6, 2017

Reference information: JCI Insight. 2017;2(7):e90721. doi:10.1172/jci.insight.90721.

\section{Introduction}

IL-5 is produced primarily by CD4+ $\mathrm{T}$ helper type 2 (Th2) cells and group 2 innate lymphoid cells (ILC2s), and is particularly important in eosinophil biology $(1,2)$. We and others have reported that the majority of IL-5-producing cells in the lung or intestine of naive or IL-33-treated mice were ILC2s, and they were responsible for maintaining and regulating eosinophils (3-6). ILC2s play important roles in allergy (7), parasitic infection (8), antiviral immunity $(9,10)$, and metabolic regulation in adipose tissues $(11-13)$. In most cases, IL-33 appears to play an important role. Although several characteristics of ILC2s have been investigated intensively, their behavior in the context of chronic inflammatory conditions or disease states, especially associated with IL-33, remains unclear.

IL-33 plays central roles in parasitic infection and allergic responses $(14,15)$. It mediates Th2 responses by enhancing Th2 cytokines, including IL-5 and IL-13. IL-33 is produced by various types of cells such as epithelial and endothelial cells $(15,16)$. IL-33 is maintained in the nucleus in a steady state and is released by necrotic cells in damaged tissue, thereby resulting in inflammation. IL-33 is therefore a proinflammatory cytokine and is likely involved in chronic inflammatory and connective tissue diseases, as well as allergies $(17,18)$. 
A

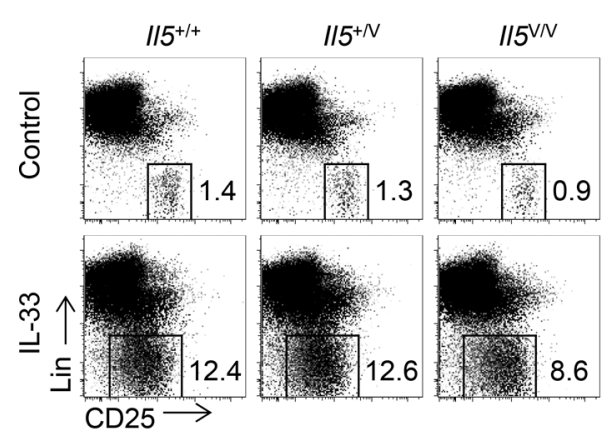

B

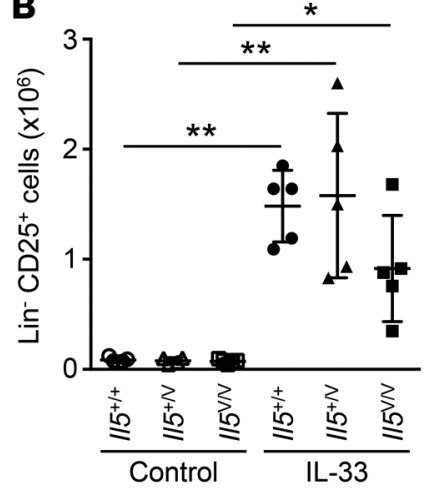

C

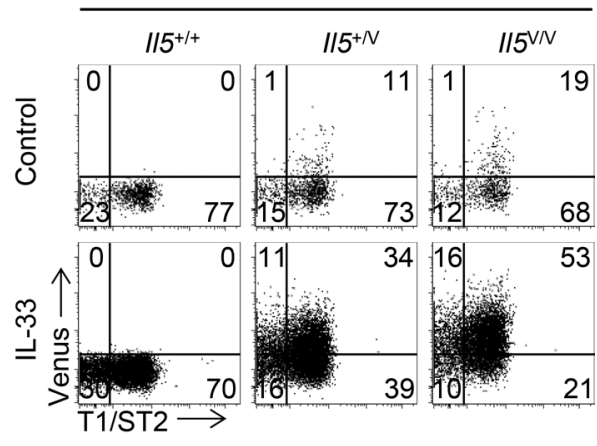

D

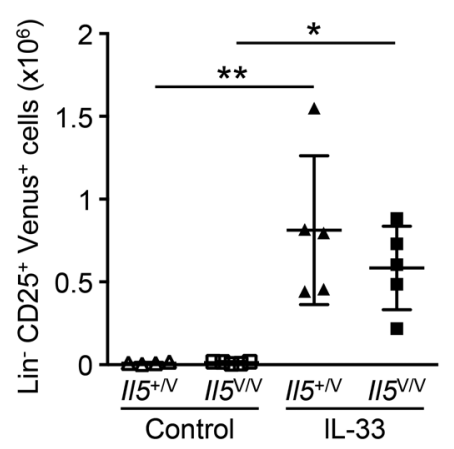

E

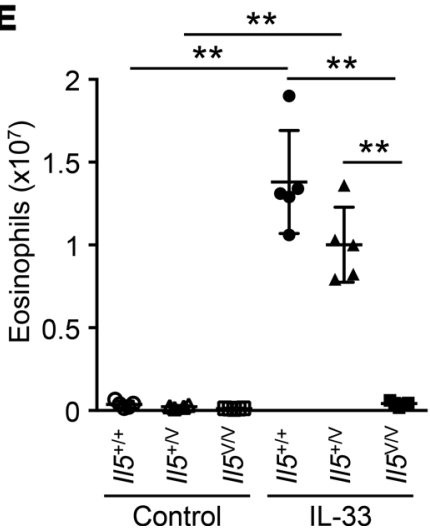

Figure 1. Expansion of IL-5-producing ILC2s and IL-5-dependent eosinophilia in the lung induced by prolonged IL-33 administration. (A) Representative flow cytometric analysis of nongranulocytes (refer to Supplemental Figure $1 \mathrm{~A}$ for flow cytometric gating) in lungs from $1 / 5^{+/+}$, $115^{+/ \mathrm{V}}$, or $/ 15^{\mathrm{V} / \mathrm{V}}$ mice ( $n=4$ or 5 per group), treated with PBS (control) or IL-33 weekly 3 times (on days 0,7 , and 14), and analyzed on day 21. Cells are shown with the expression of lineage (Lin) and CD25. Numbers show percentages of the gated cell populations. (B) Numbers of Lin-CD25+cells. Cell numbers were calculated based on the analysis from A. (C) Representative flow cytometric analysis of Lin-CD25+ cells derived from A. Cells are shown with the expression of Venus and T1/ST2. (D) Numbers of Lin-CD25+Venus ${ }^{+}$cells. Numbers were calculated based on C. (E) Numbers of eosinophils. Cell numbers were calculated based on Supplemental Figure 1E. Graph data are shown as means \pm SD. $P$ values were calculated using one-way ANOVA with Bonferroni test. Asterisks indicate statistical significance $\left.{ }^{* *} P<0.01\right)$.

In this study, we administered recombinant IL-33 to mice to investigate its potential roles in chronic inflammation and revealed that IL-33 induced hypertrophy in pulmonary arteries. Interestingly, perivascular expansion of IL-5-producing ILC2s and eosinophils was evident, and a lack of IL-5 or eosinophils resulted in amelioration of hypertrophy. This study identified IL-5-producing ILC2s as new key players in the development of arterial hypertrophy, and it reports a potential animal model to investigate the pathological process of arterial hypertrophy.

\section{Results}

Long-term IL-33 treatment induced expansion of IL-5-producing ILC2s accompanied by eosinophil recruitment. Previously, we reported that administration of IL-33 for 3 consecutive days induced a dramatic increase of IL-5-producing $\left(\right.$ Venus $^{+}$) CD $3 \varepsilon^{-}$ILC2s in the lungs (3). We assessed whether this was also the case for longterm administration. $I l 5^{+/+}, I l 5^{+/ \text {Venus }}\left(I l 5^{+/ \mathrm{v}}\right)$, and $I l 5^{\mathrm{V} / \mathrm{v}}$ (IL-5-deficient) mice were treated with 3 weekly injections of PBS or IL-33 (injections on days 0, 7, and 14). Lung cell suspensions were analyzed by flow cytometry (Figure 1 and Supplemental Figure 1; supplemental material available online with this article; https://doi.org/10.1172/jci.insight.90721DS1). The majority of Venus ${ }^{+}$cells in $\mathrm{CD}^{+} 5^{+}$nongranulocytes (flow cytometric gating is shown in Supplemental Figure 1A) were lineage negative $\left(\mathrm{Lin}^{-}\right)$and expressed CD25 (Supplemental Figure 1, B-D), confirming that the lung Venus ${ }^{+}$cells were ILC2s. Lin ${ }^{-}$CD25 $5^{+}$ILC2s were considerably expanded (Figure 1, A and B), and approximately half of these cells expressed Venus and T1/ST2 (ST2), a component of the IL-33 receptor (Figure 1, C and D). As expected, administration of 
A
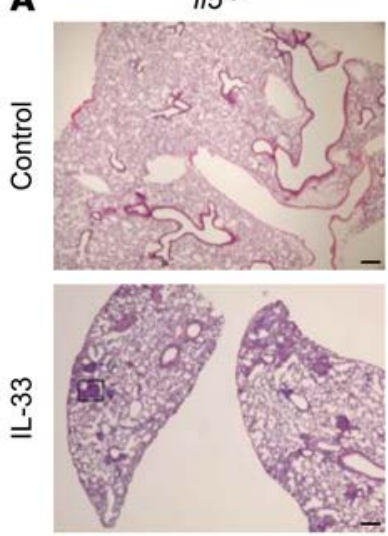

B

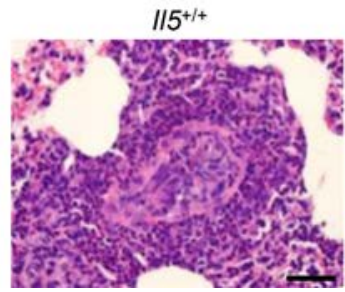

$115^{\mathrm{VN}}$
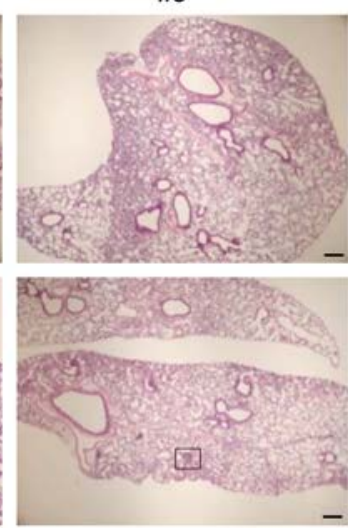

$115^{\mathrm{V} N}$

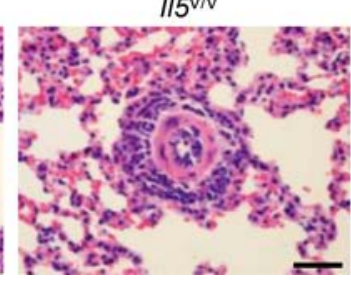

C
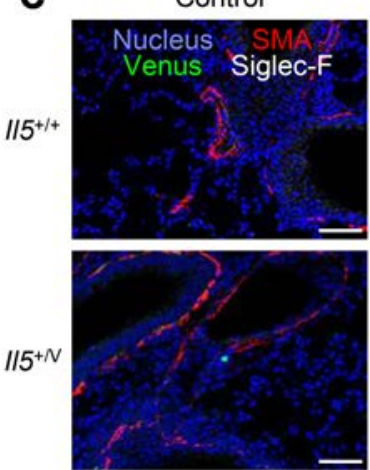

$115 \mathrm{~V} /$

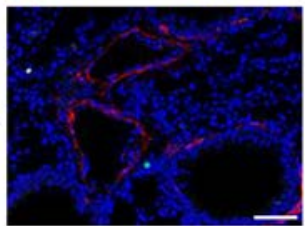

IL-33
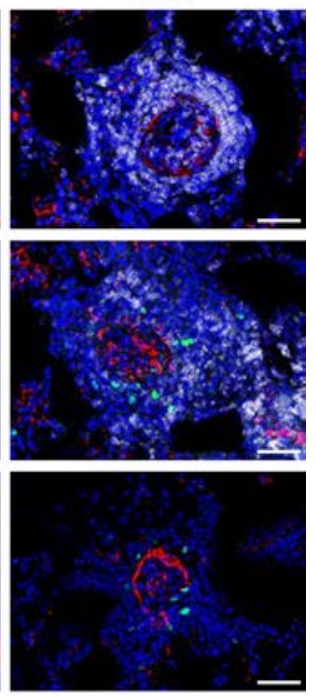

Figure 2. Arterial hypertrophy induced in IL-33-treated mice and IL-5-dependent perivascular accumulation of ILC2s and eosinophils. (A) H\&E staining of representative sections from $1 / 5^{+/+}$or $115^{\mathrm{V} / \mathrm{V}}$ mice, treated with PBS (control) or IL-33 on days 0,7 , and 14 ( $n=5$ per group). (B) High-power field of the positions enclosed by squares in A. (C) Immunofluorescent histology of lungs from $115^{+/+}$, $115^{+/ \mathrm{V}}$, or $115^{\mathrm{V} / \mathrm{V}}$ mice treated with PBS or IL-33 on days 0,7 , and 14 ( $n$ $=3$ or 4). Venus ${ }^{+}$cells (green), Siglec $-\mathrm{F}^{+}$eosinophils (white), and smooth muscle actin ${ }^{+}(\mathrm{SMA})$ smooth muscle cells (red) are shown. The nuclei are counter stained and shown in blue. Scale bars: $200 \mu \mathrm{m}$ in $\mathbf{A}$ and $50 \mu \mathrm{m}$ in $\mathbf{B}$ and $\mathbf{C}$.

IL-33 remarkably expanded Siglec- $\mathrm{F}^{+}$SSChigh eosinophils, with an approximately 50 -fold increase in $I 15^{+/+}$ and $I l 5^{+/ \mathrm{V}}$ mice (Figure $1 \mathrm{E}$ and Supplemental Figure $1 \mathrm{E}$ ). This was completely abrogated in $I l 5^{\mathrm{V} / \mathrm{v}}$ mice. There were fewer ILC2s in $I l 5^{\mathrm{v} / \mathrm{v}}$ mice than in $I l 5^{+/+}$or $I l 5^{+/ \mathrm{v}}$ mice. This is possibly due to reduced inflammation caused by IL-33 in $I l 5^{\mathrm{V} / \mathrm{v}}$ mice, as eosinophilia was almost absent in these mice.

IL-33 induced IL-5-dependent pulmonary arterial hypertrophy and perivascular eosinophilic inflammation. Histological analysis revealed severe discrete inflammation throughout the lung tissue in $\mathrm{Il}^{+/+}$mice (Figure 2A). This inflammation centered around blood vessels, and arterial hypertrophy was evident (Figure 2B). These findings were rarely observed in $I l 5^{V / V}$ mice. Perivascular IL-5-producing ILC2s were observed, and a large number of eosinophils gathered around smooth muscle actin ${ }^{+}$(SMA) smooth muscle cells in $\mathrm{Il5}^{+/+}$ and $I l 5^{+/ \mathrm{v}}$ mice, whereas such features were completely lacking in $I l 5^{\mathrm{V} / \mathrm{V}}$ mice (Figure 2C). Lung ILC2s activated by IL-25 or IL-33 produce a large amount of IL-13, leading to pulmonary fibrosis (19, 20). Consistently, long-term IL-33 administration resulted in production of IL-13, but not IL-4, from ILC2s (Supplemental Figure 2A) and induced fibrosis (Supplemental Figure 2B). Long-term systemic IL-33 treatment was also effective in increasing ILC2s and eosinophils significantly in the liver (Supplemental Figure 3, A-D). Despite the expansion of those cell populations, vascular hypertrophy or severe perivascular eosinophil accumulation was not observed in the liver (Supplemental Figure 3E). This suggests that induction of arterial hypertrophy in our system is lung specific.

The development of severe arterial hypertrophy was dependent on eosinophils, but not on T cells. Blood vessels are made up of three layers, tunica intima, tunica media, and tunica externa; these layers are primarily composed of endothelial cells, smooth muscle cells, and fibrous connective tissue, respectively. The arterial hypertrophy observed in $\mathrm{Il}^{+/+}$mice arose from advanced medial thickness and hypertrophy of the intimae (Figure 3A). We quantitatively classified the extent of hypertrophy of blood vessels referring to the HeathEdwards classification system, which is used for human samples (21) (Supplemental Figure 4). In this grading system, Grade 1 indicates hypertrophy of media; Grade 2, hypertrophy of intimae; Grade 3, advanced medial hypertrophy and progressive intimal proliferation; and Grade 4, plexiform lesions. Accordingly, 4\% of arteries were classified as Grade 1, 27\% were Grade 2, 60\% were Grade 3, and 9\% were intact in $I l 5^{+/+}$ mice (Figure $3 \mathrm{~B}$ ). In contrast, in $I l 5^{V / V}$ mice, $24 \%$ of arteries were Grade 1, $6 \%$ were Grade 2, $6 \%$ were 
A

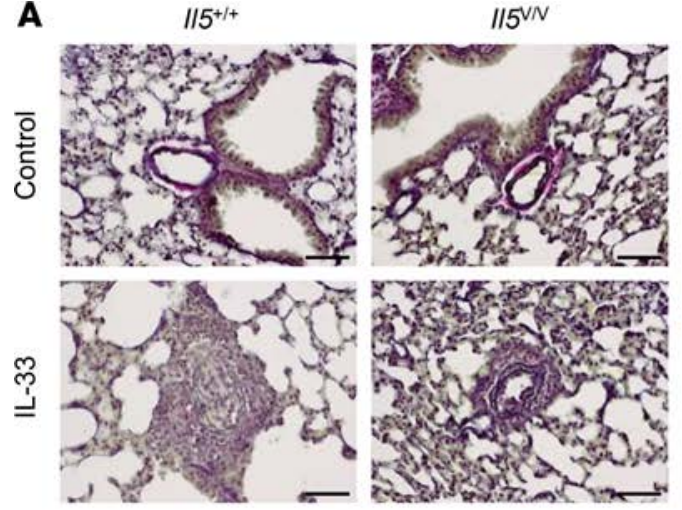

B

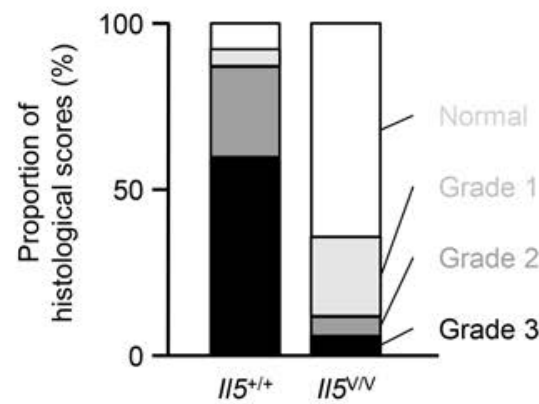

C

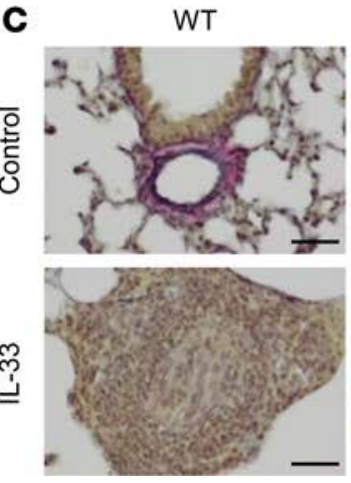

Rag $2^{-/}$

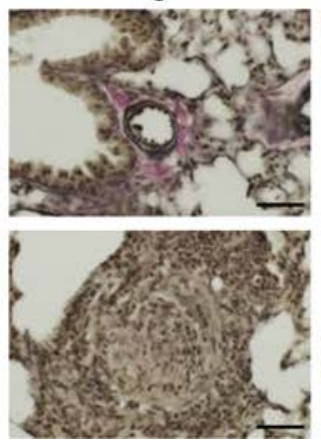

$\triangle$ dbIGATA

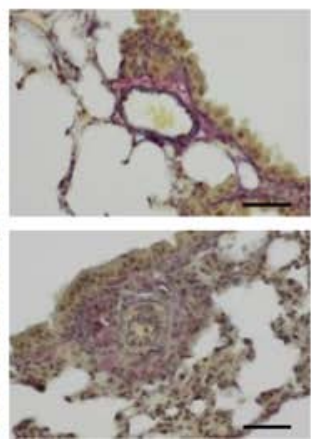

D

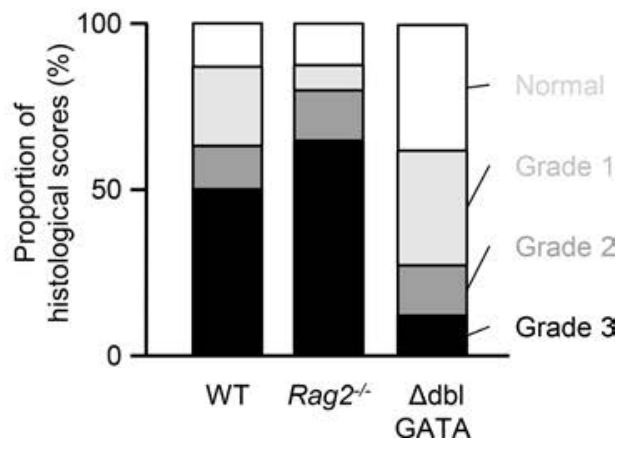

Figure 3. Advanced medial hypertrophy and progressive intimal proliferation of pulmonary arteries, which are dependent on eosinophils in IL-33treated mice. (A) Elastica van Gieson (EVG) staining of representative sections from $1 / 5^{+/+}$or $115^{\mathrm{V} / \mathrm{V}}$ mice, treated with PBS (control) or IL-33 on days 0 , 7, and 14 ( $n=4$ per group). (B) Histological scoring of arterial hypertrophy. Seventy arteries were randomly selected from IL-33-treated $/ / 5^{+/+}$or $I / 5^{\mathrm{V} / \mathrm{V}}$ mice $(n=4$ per group) and scored according to arterial conditions (Supplemental Figure 4). (C) EVG staining of representative sections from WT, Rag2-deficient, and $\triangle$ dbICATA mice treated with PBS or IL-33 on days 0,7 , and 14 ( $n=5$ for WT and $\triangle$ dblGATA mice, and $n=6$ for Rag2-deficient mice). (D) Samples of 84, 64 , or 72 randomly selected arteries from WT, Rag2-deficient, or $\Delta$ dbICATA mice, respectively, were scored. Scale bars: $50 \mu \mathrm{m}$ (A and C).

Grade 3, and 64\% were unchanged. Grade 4 was not observed. Thus, the extent of hypertrophy of pulmonary blood vessels was greatly ameliorated in mice lacking IL-5.

Next, we employed genetically engineered mice to test the hypothesis that IL-5 production by ILC2s, not by $\mathrm{T}$ cells, recruited eosinophils and that these eosinophils were involved in the development of arterial hypertrophy. WT mice, Rag2-deficient mice lacking T and B lymphocytes, and eosinophil-deficient mice ( $\triangle$ dblGATA) were treated with 3 weekly injections of IL-33, and their lungs were examined histologically. Severe arterial remodeling, such as advanced medial hypertrophy and intimal proliferation, was observed in Rag2-deficient mice, as well as in the IL-33-treated WT mice (Figure 3, C and D). However, arterial remodeling of pulmonary arteries in $\triangle$ dblGATA mice was reduced to levels similar to those found in IL-5-deficient mice treated with IL-33 (Figure 3C). We again quantified the extent of hypertrophy (Figure 3D). In WT mice, $24 \%$ of arteries were classified as Grade 1, 13\% were Grade 2, and 50\% were Grade 3, whereas in $\triangle$ dblGATA mice, $35 \%$ of arteries were classified as Grade 1, 15\% were Grade 2, and 13\% were Grade 3. This confirms that eosinophils act as effector cells. These results support the idea that IL-5-producing ILC2s, not IL-5-producing Th2 cells, were responsible for the recruitment of eosinophils that have active roles in the development of arterial hypertrophy.

A vasodilator effectively reduced IL-33-mediated arterial hypertrophy and directly suppressed expansion of ILC2. In order to investigate the possible treatment for arterial hypertrophy, we first examined the effect of anti-IL-5 antibodies. Neutralization of IL-5 with an anti-IL-5 monoclonal antibody was shown to reduce arterial hypertrophy, confirming that transient inhibition was sufficient to suppress the development of arterial hypertrophy (Figure 4, A and B). Vasodilators - including iloprost, an analogue of prostacyclin - are used clinically to treat pulmonary arterial hypertension (PAH). In order to verify our 
A
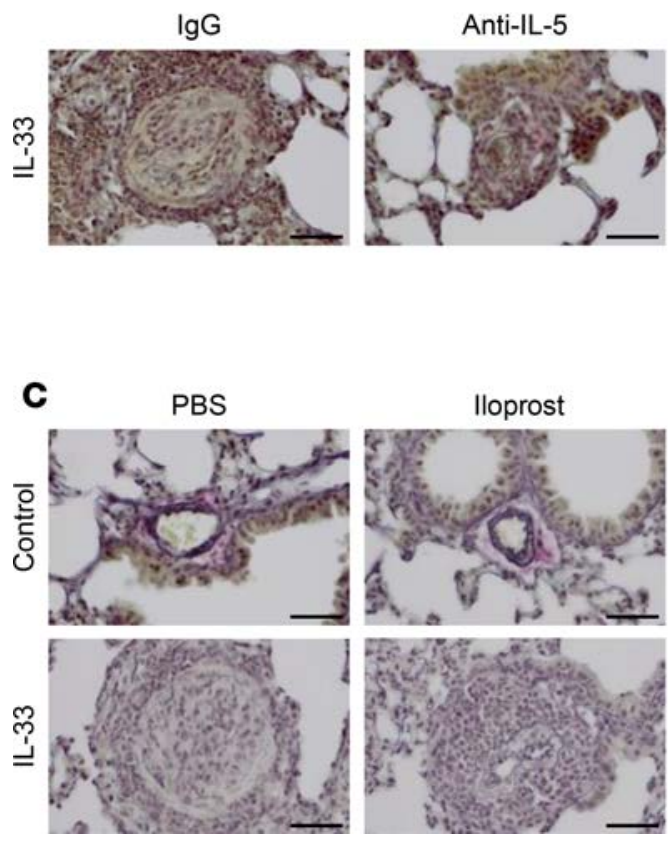

B

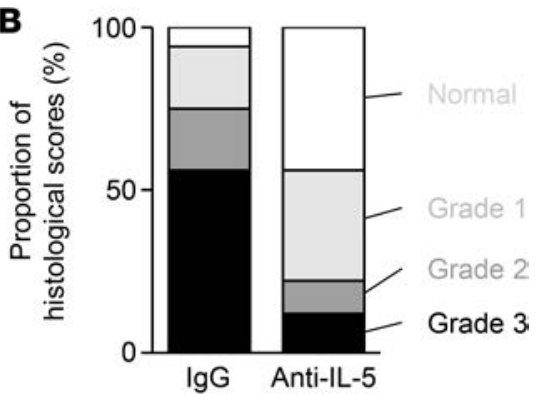

D

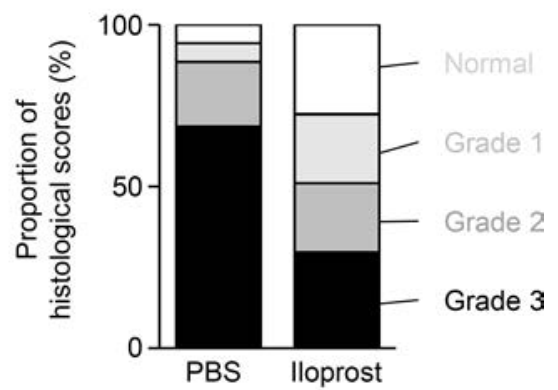

Figure 4. IL-5 neutralization or a vasodilator, iloprost, reduces arterial hypertrophy induced by IL-33. (A) Elastica van Gieson (EVG) staining of representative sections from WT mice that were treated with IL-33 and with a rat IgG antibody or an anti-IL-5 mAb ( $n=5$ per group), and analyzed on day 21. (B) Histological scoring of arterial hypertrophy. Samples of 88 or 82 arteries were randomly selected from mice treated with a control IgG or anti-IL-5 mAb, respectively. (C) EVG staining of representative sections from WT mice treated i.p. with PBS (control) or IL-33 and intratracheally with PBS or iloprost on days 0,7 , and $14(n=4$ per group). (D) Histological scoring of arterial hypertrophy. Samples of 41 or 48 randomly selected arteries from WT mice were treated i.p. with IL-33 and intratracheally with PBS or iloprost ( $n$ $=4$ per group), respectively, and examined. Scale bars: $50 \mu \mathrm{m}$ (A and C).

animal model, iloprost was used to examine whether it was also effective on animal arterial hypertrophy induced by IL-33. To evaluate the effects of iloprost, WT mice treated with PBS or IL-33 were intratracheally administered with PBS or iloprost. Lung sections were then histologically analyzed (Figure 4, C and D). The advanced medial hypertrophy and progressive intimal proliferation observed in WT mice were significantly reduced in mice treated with iloprost.

In IL-33-induced arterial hypertrophy, ILC2s that produce IL-5 and eosinophils play critical roles in the development of pulmonary arterial hypertrophy; therefore, the effect of iloprost on ILC2 proliferation and eosinophil accumulation were examined (Figure 5). The expansion of ILC2 by IL-33 administration and concomitant eosinophil recruitment were significantly decreased in mice treated with iloprost (Figure 5, A-D). We next examined the possibility that iloprost directly influences ILC2 proliferation. ILC2s were sorted from mice treated with PBS or IL-33, and mRNA expression of the prostaglandin I2 receptor (IP) was analyzed (Figure 5E). Although IL-33 downregulated the expression of IP in ILC2s, IP was still expressed in ILC2s in these conditions. Sorted ILC2s were then labeled with CFSE and cultured in the presence or absence of iloprost (Figure 5F). A greater percentage of cells cultured in the presence of iloprost remained bound to CFSE compared with those cultured in the absence of iloprost, demonstrating that iloprost was directly responsible for suppressing ILC2 proliferation.

Prolonged IL-33 administration led to the disappearance of perivascular ILC2s and eosinophils, accompanied by increased serum soluble ST2 and right ventricular hypertrophy. We next treated mice with IL-33 over a prolonged period of time to determine the impact on vascular morphology and right ventricular hypertrophy. We first analyzed eosinophils and Venus ${ }^{+}$cells surrounding arteries in $I l 5^{+/+}$or $I l 5^{V / V}$ mice, treated with 11 weekly injections of PBS or IL-33. As expected, severe arterial hypertrophy induced by IL-33 was observed in $I l 5^{+/+}$ mice, but not in $I 15^{V / V}$ mice (Supplemental Figure 5, A and B). In comparison with the group that received 3 weekly injections (Figure 2C), perivascular Venus ${ }^{+}$cells were rarely detected at this stage, and eosinophils were remarkably decreased (Figure 6A). One possible explanation for the reduced effect of IL-33 is the increased serum levels of soluble ST2, which neutralizes IL-33 activity. We therefore measured the levels of serum soluble ST2 in mice treated with IL-33 and observed elevated serum soluble ST2 in these mice (Figure 6B). These results suggest that the ILC2-eosinophil system is specifically involved in the early developmental stages of arterial hypertrophy. We finally investigated the impact of IL-33 treatment on right ventricular hypertrophy, which is one of the characteristics of pulmonary hypertension. $I l 5^{+/+}$or $I l 5^{V / V}$ mice were treated with 11 weekly injections of PBS or IL-33, and the right ventricular free wall (RV) and the left ventricle plus septum $(\mathrm{LV}+\mathrm{S})$ were weighed in order to measure the degree of right ventricular hypertrophy that was expressed as the $\mathrm{RV} /(\mathrm{LV}+\mathrm{S})$ ratio (Figure $6 \mathrm{C}) \cdot I L-5^{+/+}$mice treated with IL-33 demonstrated an increase in $\mathrm{RV} /(\mathrm{LV}+\mathrm{S})$ 
A

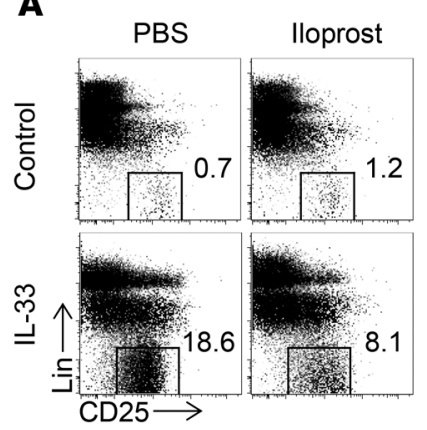

D

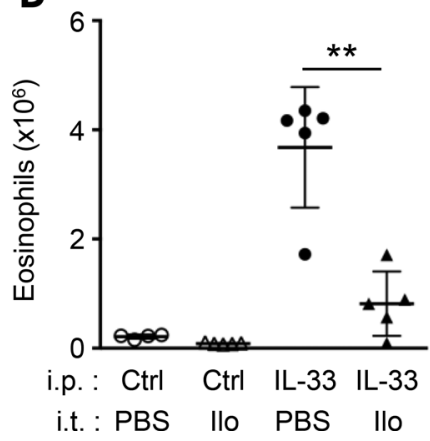

B

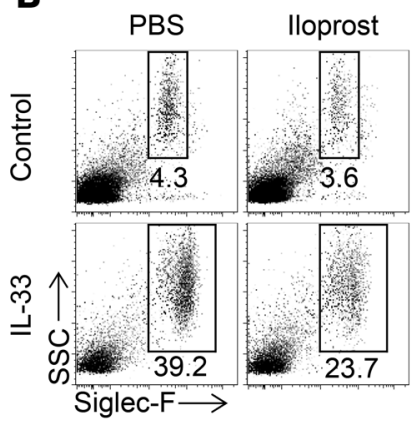

$\mathbf{E}$

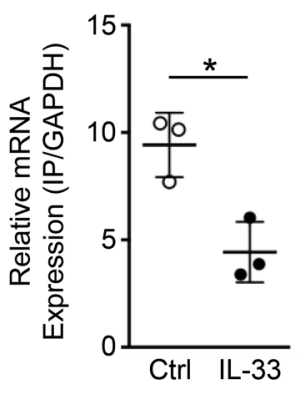

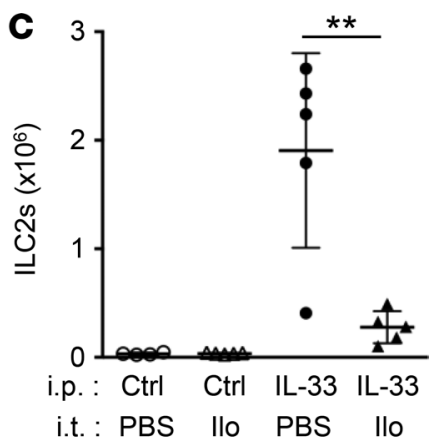

$\mathbf{F}$

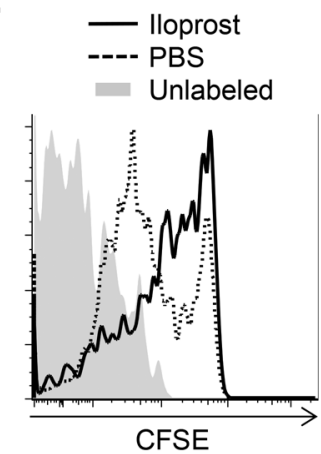

Figure 5. Iloprost directly suppresses ILC2 proliferation. (A and B) Representative flow cytometric analysis of CD25+ nongranulocytes (A) and eosinophils (B) from WT mice treated i.p. with PBS (control) or IL-33, and intratracheally with PBS or iloprost on days 0,7 , and 14 ( $n=4$ per group). Lin indicates lineage. (C and $\mathbf{D})$ Numbers of Lin-CD25+ cells (ILC2s) (C) and eosinophils (D) calculated according to flow cytometric analysis of $\mathbf{A}$ and $\mathbf{B}$, respectively. The left lobe was used for this experiment. Ctrl and Ilo indicate control and iloprost, respectively. (E) Expression of prostaglandin 12 receptor (IP) on ILC2s. CD45+Lin-CD25+ ILC2s were sorted from lung cell suspension of WT mice treated with PBS (control) or IL-33 for 3 days. Data shown are means \pm SD of three independent experiments. (F) Proliferation of purified ILC2s in the presence of iloprost in culture. Sorted ILC2s from mice treated with IL-33 for 3 days were labeled with CFSE. Unlabeled (shaded histogram) or labeled cells were cultured for 7 days with IL-33 in the presence of PBS (dotted line) or iloprost (black line). Data are representative of three independent experiments. Graph data are shown as means \pm SD. $P$ values were calculated using the two-tailed Student's $t$ test. Asterisks indicate statistical significance $\left({ }^{*} P<0.05 ;{ }^{* *} P<0.01\right)$.

ratios, whereas that increase was not found in $I I 5^{V / V}$ mice. The absolute weight of RV was increased in $I L-5^{+/+}$ mice with chronic IL-33 treatment, although it did not reach statistical significance (Figure 6D).

\section{Discussion}

In this study, we investigated the consequences of chronic administration of IL-33 and discovered an important mechanism that initiates pulmonary arterial remodeling. IL-5-producing ILC2s were found to be key factors for recruiting eosinophils to pulmonary arteries, resulting in severe arterial hypertrophy. We thus propose a new mechanism of arterial hypertrophy and predict that our study will help link chronic inflammation, in which IL-33 is involved, to the development of pulmonary arterial hypertrophy.

There is increasing experimental evidence that allergic conditions and eosinophilia are involved in the induction of arterial remodeling. In animals, persistent pulmonary allergic responses induced by house dust mite extract or ovalbumin (OVA) have been shown to trigger arterial remodeling $(22,23)$. Consistent with our results, IL-5 and eosinophils were shown to be key players in the hypertrophy of pulmonary arteries in an OVAinduced pulmonary hypertension model by means of adiponectin-deficient animals (24). In the study (24), eosinophil granule extracts promoted proliferation of smooth muscle cells. The granules released by eosinophils surrounding the arteries might have a role in arterial hypertrophy induced by IL-33. In addition, experimental Schistosoma mansoni infection can induce arterial hypertrophy $(25,26)$. In studies of schistosomiasis, Th2 cytokines, including IL- 4 and IL-13, or eosinophils were suggested to be involved in induction of arterial hypertrophy. Although IL-33 was not directly measured in all of the relevant studies, these reports support the present finding that IL-33 is a critical mediator of pulmonary arterial hypertrophy. In addition to IL-33, IL-25 
A
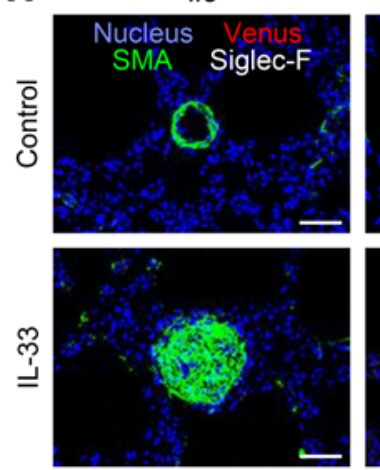

C

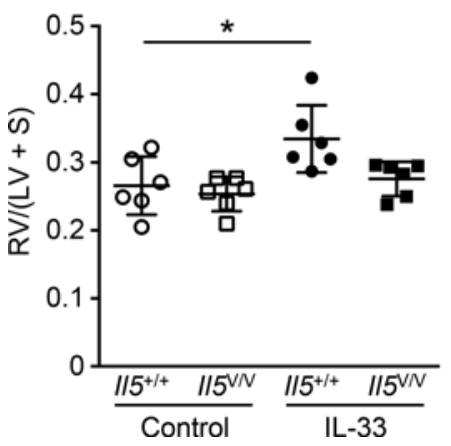

$1 / 5^{+N}$
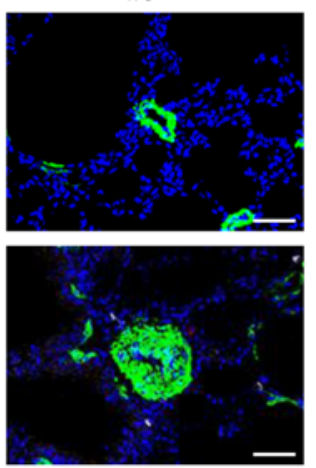

D

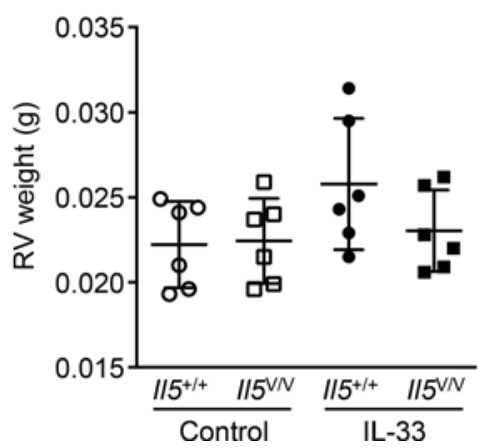

B
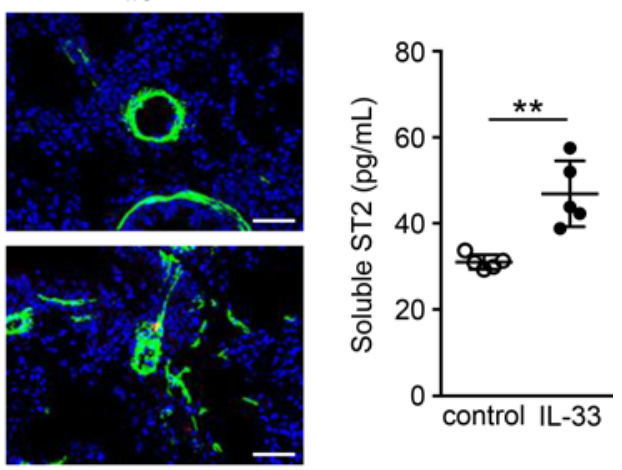

Figure 6. Prolonged chronic exposure to IL-33 resulted in disappearance of perivascular ILC2s and eosinophils, increased levels of serum soluble ST2, and right ventricular hypertrophy. (A) Immunofluorescent histology of lungs from $115^{+/+}, 115^{+/ V}$, or $115^{\mathrm{V} / \mathrm{V}}$ mice treated with 11 weekly injections of PBS (control) or IL-33 ( $n=4$ per group). Venus ${ }^{+}$cells (red) are shown with Siglec $-\mathrm{F}^{+}$eosinophils (white) and smooth muscle actin ${ }^{+}$(SMA) smooth muscle cells (green). The nuclei are visualized in blue. Scale bars: $50 \mu \mathrm{m}$. (B) Serum soluble ST2 from mice treated with PBS or IL-33 at 11 weekly intervals was measured by ELISA ( $n=5$ per group). $P$ values were calculated using the twotailed Student's $t$ test. (C and D) Comparison of right ventricular hypertrophy between $115^{+/+}$or $115^{\mathrm{V} / \mathrm{V}}$ mice treated with 11 weekly injections of PBS or IL-33 $(n=6)$. The right ventricular free wall (RV) and the left ventricle plus septum (LV + S) were weighted, and the degree of hypertrophy is shown as RV/ (LV + S) ratios (C) and the absolute RV weight (D). Graph data are shown as means \pm SD. $P$ values were calculated using one-way ANOVA with Bonferroni test. Asterisks indicate statistical significance $\left({ }^{*} P<0.05 ;{ }^{* *} P<0.01\right)$.

may induce arterial hypertrophy, as they both activate $\operatorname{ILC} 2 \mathrm{~s}$, leading to eosinophilia $(7,27)$. Overexpression of IL-25 results in perivascular eosinophilia and mild vascular hypertrophy, accompanied with airway inflammation (28). However, arterial hypertrophy is unlikely to be initiated solely by IL-5 or eosinophilia, since blood vessels in the lungs of IL-5 transgenic mice appear to be unaffected (29). This suggests that IL-33 activates other key pathways, in addition to the ILC2/eosinophil axis, to develop pulmonary vascular remodeling.

$\mathrm{PAH}$ is characterized by severe obstruction of small pulmonary arteries and concomitant high pulmonary artery pressure, leading to progressive right ventricular failure. Accumulating evidence suggests that inflammation is a key factor in inducing PAH (30). Inflammation is likely mediated by immune cells, as they are often associated with arterial blood vessel lesions (31). Studies of several animal models of human PAH, including monocrotaline- (32) and hypoxia-induced (33) models, also support the hypothesis that immune cells and inflammation are involved in PAH. The development of PAH is commonly associated with connective tissue diseases, including systemic sclerosis and systemic lupus erythematosus (34-36). Interestingly, serum levels of IL-33 are increased in these diseases $(17,18)$. Circulating IL-33 is likely to reach the lung tissue, and it ultimately leads to arterial hypertrophy, which is consistent with our observation of arterial hypertrophy following systemic IL-33 administration. More directly, serum levels of soluble ST2 in patients with PAH were found to be significantly elevated (37). The increased soluble ST2 levels may have resulted from enhanced IL-33 secretion during the early stages of development of PAH in patients. This hypothesis is supported by our results demonstrating that long-term IL-33 treatment leads to upregulation of serum soluble ST2. 
The cell type that produces the pertinent Th2 cytokines in Th2-induced arterial hypertrophy remains unclear. In OVA- or Aspergillus fumigatus antigen-induced pulmonary arterial remodeling, CD4 ${ }^{+} \mathrm{T}$ lymphocytes have been demonstrated to play a major role in the initiation of arterial hypertrophy, and Th2 cytokines are most likely produced by antigen-specific T lymphocytes (38). However, in this study, IL-33induced arterial hypertrophy was observed in Rag2-deficient mice. Consistent with our results, athymic rats can develop PAH $(39,40)$. Thus, in experimental arterial hypertrophy mediated by Th2 immune responses, there are at least two pathways: $\mathrm{T}$ cell dependent and $\mathrm{T}$ cell independent. These pathways may cooperate with or compensate for each other to induce pulmonary arterial hypertrophy, as reported previously in allergic inflammation (41).

Since ILC2s were identified, extensive studies have been conducted on regulatory molecules required for ILC2 cell biology (42). Although stimuli such as IL-25 and IL-33 have been well characterized, inhibitory molecules are relatively lesser known. We and others have demonstrated that IFN- $\gamma$ signaling negatively regulated IL-5 and IL-13 production from ILC2s and their proliferation (43-45). Lipoxin $\mathrm{A}_{4}$, derived from arachidonic acid, also inhibits IL-13 production from human ILC2s (46). A recent publication reported that an analogue of prostacyclin inhibited IL-33-induced IL-5 and IL-13 production from both mouse and human ILC2s (47). Consistently, our study also indicates prostacyclin as an inhibitory regulator because iloprost directly suppressed ILC2 proliferation. As demonstrated in the report (47), IP-mediated signaling could be a new target for regulating ILC2s in the treatment of allergic diseases.

Our study reveals a previously unrecognized role of ILC2s in pulmonary arterial hypertrophy, and understanding IL-33-induced arterial hypertrophy will provide useful information about the development of pulmonary arterial hypertrophy, possibly leading to $\mathrm{PAH}$.

\section{Methods}

Mice. C57BL/6 WT mice were purchased from Japan SLC. IL-5/Venus knock-in mice were generated as previously reported (3). Rag2-deficient mice (48) were provided by Frederick W. Alt (Howard Hughes Medical Institute, Children's Hospital Boston, Department of Genetics, Harvard Medical School, Boston, Massachusetts, USA). $\triangle$ dblGATA mice (49) were obtained from The Jackson Laboratory. These genetically engineered mice were backcrossed at least 10 generations to C57BL/6 mice. All mice were maintained under specific pathogen-free conditions in the animal facility of the University of Toyama. Mice used were male and 7-11 weeks of age. For systemic administration of IL-33 (R\&D Systems), mice were i.p. injected with $400 \mathrm{ng}$ of recombinant IL-33 on days 0,7 , and 14, and were euthanized on day 21 . In some experiments, IL-33 (400 ng) was injected i.p. at weekly intervals for 11 consecutive weeks. To neutralize IL-5, a single bolus of $100 \mu \mathrm{g}$ of anti-IL-5 mAb (NC17) $(50,51)$ or a control rat IgG antibody (Sigma-Aldrich) was i.p. administered for 3 consecutive days before and at each weekly IL-33 administration (days $-2,-1,0,5$, $6,7,12,13$, and 14). A $2 \mu \mathrm{g}$ dose of iloprost (Cayman Chemical) was intratracheally administered on days 0,7 , and 14 during systemic administration of IL-33.

Preparation of lung cells. Lungs were perfused with $5 \mathrm{ml} \mathrm{PBS}$, harvested, and all lobes were minced in $1 \mathrm{ml}$ of RPMI-1640 (Invitrogen) with 10\% FBS in the presence of $1 \mathrm{mg} / \mathrm{ml}$ collagenase A (Roche Diagnostics) and $100 \mu \mathrm{g} / \mathrm{ml}$ DNase I (Sigma-Aldrich). After incubation for 1 hour at $37^{\circ} \mathrm{C}$ in a $\mathrm{CO}_{2}$ incubator, minced lungs were mashed on a $70 \mu$ m nylon mesh (BD Biosciences) with a plunger, resulting in a lung cell suspension.

Antibodies and reagents for flow cytometry and immunofluorescent histology. Antibodies used for flow cytometry included the following: anti-mouse B220 (catalog 561878), CD11c (catalog 561044), CD19 (catalog 561736), CD45 (catalog 561868), Gr-1 (catalog 561084), and Siglec-F (catalog 562068) purchased from BD Biosciences; CD3ع (catalog 12-0031-81), CD25 (catalog 17-0251-81), FceRIa (catalog 12-5898-81), Mac1 (catalog 12-0112-81), and TER-119 (catalog 12-5921-81) purchased from eBioscience; CD49b (catalog 108907) and NK1.1 (catalog 108707) purchased from BioLegend; and ST2 (catalog 101001B) purchased from MD Bioscience. Fc $\gamma$ Rs were blocked with anti-mouse Fc $\gamma$ R (catalog 553141, BD Biosciences). Lineage markers were stained with a cocktail of antibodies for mice, including B220, CD3e, CD11c, CD19, CD49b, FceRIa, Gr-1, Mac-1, NK1.1, and TER-119. Flow cytometry was performed on a FACSCanto II (BD Biosciences). Dead cells were gated out by 7-AAD staining (BD Biosciences). FlowJo software was used for analysis. The antibodies used for immunofluorescent histology included anti-mouse Siglec-F (cata$\log 562680$ ) purchased from BD Biosciences and anti-SMA (catalog ab184675 or ab8207) purchased from Abcam. Polyclonal rabbit anti-GFP antibodies (catalog 598) were purchased from Medical \& Biological 
Laboratories. Unlabeled primary antibodies were visualized with secondary anti-rabbit IgG conjugated with Alexa 488 or 568 (catalog A-11034 or A-11011, respectively, Invitrogen), or anti-mouse IgG conjugated with Alexa 568 (catalog A-11004, Invitrogen). Hoechst 33342 (Invitrogen) was used to stain nuclei.

IHC. For immunofluorescent histology, lungs were fixed with $4 \%$ paraformaldehyde, embedded in OCT compound (Sakura Finetek), and frozen at $-80^{\circ} \mathrm{C}$. Frozen samples were cut into $30-\mu \mathrm{m}$ slices using a cryostat (Leica Microsystems). Sections were incubated with TSA blocking reagent (PerkinElmer), washed, and incubated with primary antibodies at the respective manufacturer's recommended concentration overnight at $4^{\circ} \mathrm{C}$. Subsequently, sections were washed and incubated with secondary antibodies. Finally, samples were mounted in Fluoromount (Diagnostic BioSystems). Individual fluorescent images were acquired using a TCS SP5 confocal microscope and imaging system (Leica Microsystems), and trimmed and overlaid with Photoshop software (Adobe Systems). For H\&E and Elastica van Gieson (EVG) staining, lungs were fixed with $4 \%$ paraformaldehyde and embedded in paraffin. Samples were cut into 4- $\mu \mathrm{m}$ slices using a microtome (Sakura Finetek). After deparaffinization, sections were processed for staining. Images were acquired using a D-33E digital camera (Olympus).

Cell sorting and culture. Lung cells were harvested from WT mice administered with IL-33 or PBS for 3 consecutive days. Whole cells were gated on $\mathrm{CD} 45^{+}$cells, and $\mathrm{Lin}^{-} \mathrm{CD} 25^{+}$cells were sorted as ILC2s. Sorting purity (mean \pm SD) for PBS- or IL-33-treated ILC2s were $98.2 \% \pm 1.6 \%$ or $98.6 \% \pm$ $1.2 \%$, respectively. For cell culture, RPMI-1640 medium supplemented with $10 \%$ FBS, $50 \mu \mathrm{M} 2$-mercaptoethanol (Life Technologies), $100 \mathrm{U} / \mathrm{ml}$ penicillin (Life Technologies), and $100 \mu \mathrm{g} / \mathrm{ml}$ streptomycin (Life Technologies) was used. Sorted ILC2s were labeled with CFSE, and 20,000 cells per well were cultured in the presence of IL-33 $(10 \mathrm{ng} / \mathrm{ml})$ for 6 days. Flow cytometric analysis was performed on day 7 .

Real time PCR. Total RNA from sorted ILC2s was purified using the RNeasy Mini Kit (Qiagen). cDNA was synthesized using the High Capacity RNA-to-cDNA Kit (Applied Biosystems) and prepared for real time PCR using the Applied Biosystems StepOne Plus Real Time PCR System. Taqman primers and probes for Gapdh (Mm99999915_g1) and Ptgir (IP) (Mm00801939m1) were purchased from Applied Biosystems.

ELISA. Levels of serum soluble ST2 in plasma were measured using a mouse ST2 ELISA kit (R\&D Systems). Plasma was collected from WT mice, treated with PBS or IL-33 eleven times at weekly intervals.

Statistics. $P$ values were calculated using the two-tailed Student's $t$ test or Graphpad Prism 6 software for multiple comparisons.

Study approval. All experiments were approved by the Animal Experiment Committee of the University of Toyama and performed in accordance with the Guidelines for the Care and Use of Laboratory Animal of the University of Toyama.

\section{Author contributions}

MI planned the project, performed experiments, and wrote the manuscript. K. Tsuneyama designed the project, performed histological examination, and interpreted the results. FK helped cell sorting and liver analysis. MK and JF analyzed histological samples. SN and MA supervised IL-33 and eosinophil work. YN, ST, and K. Takatsu supervised IL-5 and ILC2 work, and revised the manuscript prior to submission.

\section{Acknowledgments}

We are grateful to Toyama Prefecture (Japan) for supporting our laboratory. We thank the members of our laboratories for their assistance. This work was supported by JSPS KAKENHI (grant 24390119 to K. Takatsu and grant 26860319 to MI), the Nagao Memorial Fund, and the Grant for Joint Research Project of The Institute of Medical Science, The University of Tokyo (Tokyo, Japan) (Grant number 2014-239).

Address correspondence to: Masashi Ikutani, Department of Immune Regulation, The Research Centre for Hepatitis and Immunology, National Centre for Global Health and Medicine, 1-7-1 Kohnodai, Ichikawa-shi, Chiba 272-8516, Japan. Phone: 81.47.375.4761; E-mail: 1bmikutani@hospk.ncgm.go.jp. Or to: Kiyoshi Takatsu, Department of Immunobiology and Pharmacological Genetics, Graduate School of Medicine and Pharmaceutical Science for Research, University of Toyama, 2630 Sugitani, Toyama-shi, Toyama 930-0194, Japan. Phone: 81.76.434.7673; E-mail: takatsuk@med.u-toyama.ac.jp. 
1. Takatsu K, Nakajima H. IL-5 and eosinophilia. Curr Opin Immunol. 2008;20(3):288-294.

2. Takatsu K. Interleukin-5 and IL-5 receptor in health and diseases. Proc Jpn Acad, Ser B, Phys Biol Sci. 2011;87(8):463-485.

3. Ikutani M, et al. Identification of innate IL-5-producing cells and their role in lung eosinophil regulation and antitumor immunity. J Immunol. 2012;188(2):703-713.

4. Moro K, et al. Innate production of $\mathrm{T}(\mathrm{H}) 2$ cytokines by adipose tissue-associated c-Kit(+)Sca-1(+) lymphoid cells. Nature. 2010;463(7280):540-544.

5. Neill DR, et al. Nuocytes represent a new innate effector leukocyte that mediates type-2 immunity. Nature. 2010;464(7293):1367-1370.

6. Price AE, et al. Systemically dispersed innate IL-13-expressing cells in type 2 immunity. Proc Natl Acad Sci USA. 2010;107(25):11489-11494.

7. Klein Wolterink RG, Hendriks RW. Type 2 innate lymphocytes in allergic airway inflammation. Curr Allergy Asthma Rep. 2013;13(3):271-280.

8. Yasuda K, et al. Contribution of IL-33-activated type II innate lymphoid cells to pulmonary eosinophilia in intestinal nematodeinfected mice. Proc Natl Acad Sci USA. 2012;109(9):3451-3456.

9. Monticelli LA, et al. Innate lymphoid cells promote lung-tissue homeostasis after infection with influenza virus. Nat Immunol. 2011;12(11):1045-1054.

10. Chang YJ, et al. Innate lymphoid cells mediate influenza-induced airway hyper-reactivity independently of adaptive immunity. Nat Immunol. 2011;12(7):631-638.

11. Molofsky AB, et al. Innate lymphoid type 2 cells sustain visceral adipose tissue eosinophils and alternatively activated macrophages. JExp Med. 2013;210(3):535-549.

12. Lee MW, et al. Activated type 2 innate lymphoid cells regulate beige fat biogenesis. Cell. 2015;160(1-2):74-87.

13. Brestoff JR, et al. Group 2 innate lymphoid cells promote beiging of white adipose tissue and limit obesity. Nature. 2015;519(7542):242-246.

14. Cayrol C, Girard JP. IL-33: an alarmin cytokine with crucial roles in innate immunity, inflammation and allergy. Curr Opin Immunol. 2014;31:31-37.

15. Ohno T, Morita H, Arae K, Matsumoto K, Nakae S. Interleukin-33 in allergy. Allergy. 2012;67(10):1203-1214.

16. Liew FY, Pitman NI, McInnes IB. Disease-associated functions of IL-33: the new kid in the IL-1 family. Nat Rev Immunol. 2010;10(2):103-110.

17. Oboki K, Ohno T, Kajiwara N, Saito H, Nakae S. IL-33 and IL-33 receptors in host defense and diseases. Allergol Int. 2010;59(2):143-160.

18. Pei C, Barbour M, Fairlie-Clarke KJ, Allan D, Mu R, Jiang HR. Emerging role of interleukin-33 in autoimmune diseases. Immunology. 2014;141(1):9-17.

19. Li D, et al. IL-33 promotes ST2-dependent lung fibrosis by the induction of alternatively activated macrophages and innate lymphoid cells in mice. J Allergy Clin Immunol. 2014;134(6):1422-1432.e11.

20. Hams E, et al. IL-25 and type 2 innate lymphoid cells induce pulmonary fibrosis. Proc Natl Acad Sci USA. 2014;111(1):367-372

21. Heath D, Edwards JE. The pathology of hypertensive pulmonary vascular disease; a description of six grades of structural changes in the pulmonary arteries with special reference to congenital cardiac septal defects. Circulation. 1958;18(4 Part 1):533-547.

22. Törmänen KR, Uller L, Persson CG, Erjefält JS. Allergen exposure of mouse airways evokes remodeling of both bronchi and large pulmonary vessels. Am J Respir Crit Care Med. 2005;171(1):19-25.

23. Rydell-Törmänen K, Johnson JR, Fattouh R, Jordana M, Erjefält JS. Induction of vascular remodeling in the lung by chronic house dust mite exposure. Am J Respir Cell Mol Biol. 2008;39(1):61-67.

24. Weng M, Baron DM, Bloch KD, Luster AD, Lee JJ, Medoff BD. Eosinophils are necessary for pulmonary arterial remodeling in a mouse model of eosinophilic inflammation-induced pulmonary hypertension. Am J Physiol Lung Cell Mol Physiol. 2011;301(6):L927-L936.

25. Crosby A, et al. Pulmonary vascular remodeling correlates with lung eggs and cytokines in murine schistosomiasis. Am J Respir Crit Care Med. 2010;181(3):279-288.

26. Graham BB, et al. Schistosomiasis-induced experimental pulmonary hypertension: role of interleukin-13 signaling. Am J Pathol. 2010;177(3):1549-1561

27. Licona-Limón P, Kim LK, Palm NW, Flavell RA. TH2, allergy and group 2 innate lymphoid cells. Nat Immunol. 2013;14(6):536-542

28. Fort MM, et al. IL-25 induces IL-4, IL-5, and IL-13 and Th2-associated pathologies in vivo. Immunity. 2001;15(6):985-995.

29. Lee JJ, et al. Interleukin-5 expression in the lung epithelium of transgenic mice leads to pulmonary changes pathognomonic of asthma. J Exp Med. 1997;185(12):2143-2156.

30. Price LC, et al. Inflammation in pulmonary arterial hypertension. Chest. 2012;141(1):210-221.

31. Tuder RM, Groves B, Badesch DB, Voelkel NF. Exuberant endothelial cell growth and elements of inflammation are present in plexiform lesions of pulmonary hypertension. Am J Pathol. 1994;144(2):275-285.

32. Gomez-Arroyo JG, et al. The monocrotaline model of pulmonary hypertension in perspective. Am J Physiol Lung Cell Mol Physiol. 2012;302(4):L363-L369.

33. Stenmark KR, Fagan KA, Frid MG. Hypoxia-induced pulmonary vascular remodeling: cellular and molecular mechanisms. Circ Res. 2006;99(7):675-691.

34. Mukerjee D, et al. Prevalence and outcome in systemic sclerosis associated pulmonary arterial hypertension: application of a registry approach. Ann Rheum Dis. 2003;62(11):1088-1093.

35. Hachulla E, et al. Early detection of pulmonary arterial hypertension in systemic sclerosis: a French nationwide prospective multicenter study. Arthritis Rheum. 2005;52(12):3792-3800.

36. Fagan KA, Badesch DB. Pulmonary hypertension associated with connective tissue disease. Prog Cardiovasc Dis. 2002;45(3):225-234

37. Carlomagno G, et al. Serum soluble ST2 and interleukin-33 levels in patients with pulmonary arterial hypertension. Int J Cardiol. 2013;168(2):1545-1547. 
38. Daley E, et al. Pulmonary arterial remodeling induced by a Th2 immune response. J Exp Med. 2008;205(2):361-372.

39. Miyata M, Sakuma F, Ito M, Ohira H, Sato Y, Kasukawa R. Athymic nude rats develop severe pulmonary hypertension following monocrotaline administration. Int Arch Allergy Immunol. 2000;121(3):246-252.

40. Taraseviciene-Stewart L, et al. Absence of T cells confers increased pulmonary arterial hypertension and vascular remodeling Am J Respir Crit Care Med. 2007;175(12):1280-1289.

41. Kamijo S, et al. IL-33-mediated innate response and adaptive immune cells contribute to maximum responses of protease allergen-induced allergic airway inflammation. J Immunol. 2013;190(9):4489-4499.

42. Klose CS, Artis D. Innate lymphoid cells as regulators of immunity, inflammation and tissue homeostasis. Nat Immunol. 2016;17(7):765-774

43. Kudo F, et al. Interferon- $\gamma$ constrains cytokine production of group 2 innate lymphoid cells. Immunology. 2016;147(1):21-29.

44. Moro K, et al. Interferon and IL-27 antagonize the function of group 2 innate lymphoid cells and type 2 innate immune responses. Nat Immunol. 2016;17(1):76-86.

45. Molofsky AB, et al. Interleukin-33 and Interferon- $\gamma$ Counter-Regulate Group 2 Innate Lymphoid Cell Activation during Immune Perturbation. Immunity. 2015;43(1):161-174.

46. Barnig C, et al. Lipoxin A4 regulates natural killer cell and type 2 innate lymphoid cell activation in asthma. Sci Transl Med. 2013;5(174):174ra26.

47. Zhou W, et al. Prostaglandin I2 Signaling and Inhibition of Group 2 Innate Lymphoid Cell Responses. Am J Respir Crit Care Med. 2016;193(1):31-42.

48. Shinkai Y, et al. RAG-2-deficient mice lack mature lymphocytes owing to inability to initiate V(D)J rearrangement. Cell. 1992;68(5):855-867.

49. Yu C, et al. Targeted deletion of a high-affinity GATA-binding site in the GATA-1 promoter leads to selective loss of the eosinophil lineage in vivo. J Exp Med. 2002;195(11):1387-1395.

50. Hitoshi Y, Yamaguchi N, Korenaga M, Mita S, Tominaga A, Takatsu K. In vivo administration of antibody to murine IL-5 receptor inhibits eosinophilia of IL-5 transgenic mice. Int Immunol. 1991;3(2):135-139.

51. Harada N, et al. Production of a monoclonal antibody useful in the molecular characterization of murine T-cell-replacing factor/B-cell growth factor II. Proc Natl Acad Sci USA. 1987;84(13):4581-4585. 